\title{
Effects of Radially Sheared Electric Field on Turbulence Reduction Analyzed with End-Loss Analysing Systems
}

\author{
Mafumi HIRATA, Teruji CHO, Junko KOHAGURA, Tomoharu NUMAKURA, \\ Serina KIMINAMI, Marie ITO, Naoyuki MORIMOTO, Katsuaki HIRAI, \\ Tooru YAMAGISHI, Toshinori IKUNO, Shohei NAMIKI, Yoshiaki MIYATA, \\ Ryutaro MINAMI, Kazuo OGURA ${ }^{1)}$, Takashi $\mathrm{KONDOH}^{2)}$, Tsuyoshi KARIYA, \\ Tsuyoshi IMAI and Syoichi MIYOSHI \\ Plasma Research Center, University of Tsukuba, Ibaraki 305-8577, Japan \\ ${ }^{1)}$ Graduate School of Science and Technology, Niigata University, Niigata 950-2181, Japan \\ 2) Japan Atomic Energy Agency, Naka, Ibaraki 311-0193, Japan
}

(Received 9 December 2006 / Accepted 17 April 2007)

\begin{abstract}
Mirror devices, having open-ended regions, provide intrinsic important advantages in terms of the control of radial-potential or $E_{r}$ shear profiles on the basis of axial particle loss control for ambipolar potential formation. From the viewpoint of the mechanism investigation of radially localized transport-barrier formation, an idea of off-axis-resonant electron-cyclotron heating $(\mathrm{ECH})$ is proposed and applied in the tandem mirror GAMMA 10, by the use of the aforementioned intrinsic advantage of mirror devices. The off-axis ECH produces a cylindrical layer with energetic electrons and facilitates the formation for a localized bumped ambipolar potential with a strong shear of radial electric fields $E_{r}$. Remarkable effects of $d E_{r} / d r$ on the suppression of turbulent fluctuations with plasma confinement improvement are found by the use of signals of end-loss currents flowing from the central cell as well as the central-cell soft $\mathrm{x}$ ray. In association with the reduction of the fluctuations due to strong $E_{r}$ shear formation, increases in ion and electron temperatures are found.
\end{abstract}

(C) 2007 The Japan Society of Plasma Science and Nuclear Fusion Research

Keywords: tandem mirror, radially sheared electric field, electron-cyclotron heating, transport-barrier, end-loss analyzer

DOI: $10.1585 /$ pfr.2.S1077

\section{Introduction}

For the purpose of improving confinement of fusion plasmas, one of the most critical issues is to understand common physics essentials lying in the formation of transverse transport barrier or $\mathrm{H}$ mode found in various types of devices individually [1,2]. From theoretical predictions, these phenomena are believed to have close relations with potential and/or electric field formation [1-3].

From the viewpoint of investigating the contributions of radially sheared electric fields $E_{r}$ to the issue, openended mirror devices [4-15] have advantageous and inherent characteristics of an experimental ease of controls of a radial potential profile. Recently, intermittent turbulent vortex structures and effects of their suppression by strongly sheared plasma rotation were observed in the GAMMA 10 tandem mirror [5-9]. The suppression of turbulence and the associated significant reduction in crossfield transport in GAMMA 10 show behaviors that are similar to those seen for L-H transitions in tokamaks. Mirror devices, having open-ended regions, provide intrinsic important advantages in terms of the control of radialpotential or sheared $\boldsymbol{E}_{r} \times \boldsymbol{B}$ rotation profiles on the basis of

author'se-mail: mafumi@prc.tsukuba.ac.jp axial particle loss control for ambipolar potential formation.

Such a control of $E_{r}$ in a mirror is easily carried out on the basis of driving axial energetic-electron flows by using electron cyclotron heating (ECH) [16-20] into open-ended regions along lines of magnetic force. The loss of ECH accelerated (or pumped out) electrons as compared with background unaffected ions results in positive potentials (i.e., Poisson's law).

In this paper, the effects of actively controlled radial potential or $E_{r}$ shear flow profiles on the basis of off-axis resonant ECH are demonstrated. Formation of an alternative radial transport barrier, which has similar properties to ITB in toroidal plasmas $[1,2]$, has been observed in the vicinity of the strong shear flow layer with a high vorticity. This experiment denotes suppression of intermittent turbulent vortex-like structures near the layer in the central cell with a transverse energy-transport improvement. This new method for an active control of the formation of radial energy transport barrier is visually highlighted by the suppression of intermittent broadband fluctuations or vortexlike structures in L-mode like turbulent plasmas, which are produced by ion cyclotron heating ( $\mathrm{ICH})$ with ion temperatures $T_{i} \sim 4 \mathrm{keV}$ for the off-axis ECH target. 


\section{Experimental Apparatus}

GAMMA 10 , which is a minimum- $B$ anchored tandem mirror with outboard axisymmetric plug and barrier cells [3-12, 14-19], has an axial length $\left(L_{z}\right)$ of $27 \mathrm{~m}$, and the vacuum vessel of $150 \mathrm{~m}^{3}$. In the central cell $\left(L_{z}=6 \mathrm{~m}\right.$, limiter diameter of $36 \mathrm{~cm}$, and magnetic fields $B_{z}=B_{m}$ at the midplane of $0.405 \mathrm{~T}$ with a mirror ratio $R_{m}$ of 5.2.), Ion cyclotron heating $(\mathrm{ICH} ; 30 \mathrm{~kW}$ at $6.36 \mathrm{MHz}$ for centralcell hot-ion production, and $30 \mathrm{~kW}$ at 9.9 or $10.3 \mathrm{MHz}$ for anchor stabilization) is applied. The plug and barrier cells are axisymmetric mirrors with $L_{z}=2.5 \mathrm{~m}, B_{m}=0.497 \mathrm{~T}$, and $R_{m}=6.2$ for standard operations. In the transport barrier experiments, $4.75 \%$ higher $B_{m}(0.519 \mathrm{~T})$ is applied in the barrier cell for off-axis resonant $\mathrm{ECH}$ to produce the cylindrical energetic electron layer. Gyrotron power of $120 \mathrm{~kW}$ at $28 \mathrm{GHz}$ is injected into a single (east) barrier cell alone. No additional ECH is applied, thereby simplifying the experimental situations (see Sec. 3).

Various fluctuation diagnostics including a movable microwave interferometer, the Fraunhofer-diffraction method, two sets of our developed fifty-channel soft X-ray tomography detectors using microchannel plates (MCP) [16-21] in the central-cell midplane, several original semiconductor or metal collector detectors in various cells for $T_{e}$ and $T_{i}$ diagnostics [22-28], eight Langmuir probes for wave phasing and coherence diagnostics [6], heavy-ion $\left(\mathrm{Au}^{0}\right)$ beam probes (HIBP) and eight sets of ion-energyspectrometer (IES) arrays [7, 29-32], and simultaneous potential diagnostics with HIBP and IES show consistent characteristics (see below). Energetic electron currents are observed with a radially movable conventional end-loss analyzer (ELA) and ELA arrays [7,29] at the west end.

\section{Effect of the Formation of Energet- ic-Electron Layer with $E_{r}$ Shear}

From the viewpoint of the mechanism investigation of radially localized transport-barrier formation with relation to toroidal devices, an idea of off-axis-resonant (barrier) $\mathrm{ECH}$ is proposed and applied (Fig. 1(a)) by the use of the aforementioned intrinsic advantage of mirror devices. This allows for flexible experiments regarding relations between shear profiles and reduction in turbulencedriven radial losses due to $\mathrm{ECH}$ power-profile control. Radial profile of these electrons and electron energy spectrum are observed with a radially movable ELA at the west end (Fig. 1). The radial positions are mapped along the magnetic flux tubes into the central-cell plasma radius $r_{c}$. The cylindrical layer is centered near $r_{c}=5.5 \mathrm{~cm}$ with halfwidth of approximately $1.5 \mathrm{~cm}$. The energetic electrons have energy about $2 \mathrm{keV}$ and have a population of the order of $10^{-5}$ with respect to the total density in the layer. Thus, no beta-value effects and negligible slowing down plasma heatings are anticipated from the parameters. The layer is axially formed throughout the device; i.e., energetic electrons present in the layer due to the (east) barrier-
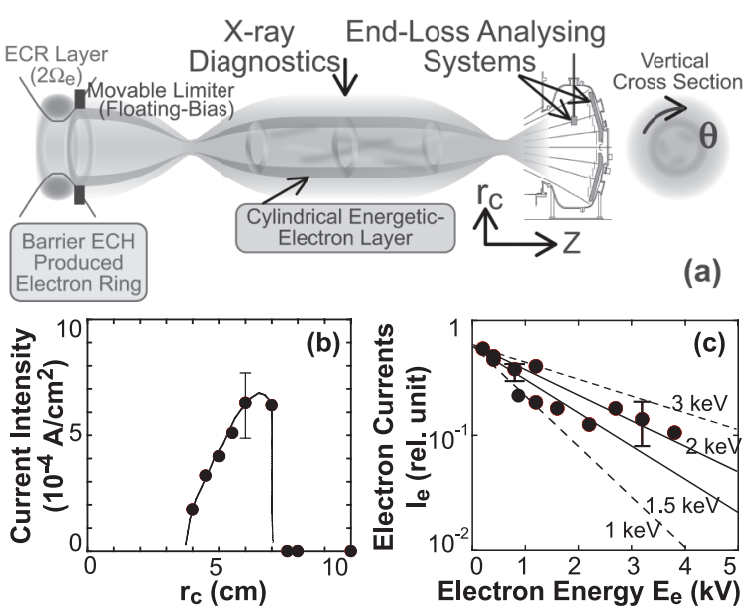

Fig. 1 (a) Schematic drawing of experimental configuration for cylindrical energetic-electron layer formation by the use of off-axis barrier ECH in GAMMA 10. X-ray imaging systems are installed in the central cell. (b) Radial energetic-electron current profile and (c) energeticelectron energy spectrum during the barrier $\mathrm{ECH}$ are measured with the end-loss analyzing systems in the end region.

cell ECH flow through the central cell and escape into the open-ended region. As described above, the loss of the energetic electrons leads to create a positively bumped potential enhancement located in the cylindrical layer with $E_{r}$ shear formation.

To investigate the effects of the formation of this energetic-electron layer, Fourier analyses of the centralcell soft $x$-ray brightness and the ion signals with IES arrays are presented (Figs. 2, 3). Fourier amplitudes of $x$ rays at $r_{c}=2.7$ and $10 \mathrm{~cm}$ without and with the energeticelectron-layer formation are shown in Figs. 2 (a)-2(d), respectively. Turbulent spectra at any $r_{c}$ without the layer [Figs. 2(a), 2(c)], and at $r_{c}$ inside the layer [Fig. 2(b)] are observed. The turbulence is, however, significantly reduced in Fig. 2(d) with the layer formation. Similar fluctuation dependence is observed in the ion currents from the ELA array. That is, turbulent spectra are observed at $r_{c}=2.6$ and $8.3 \mathrm{~cm}$ without the layer [Figs. $3(\mathrm{a}), 3(\mathrm{c})$ ], and at $r_{c}=2.6$ inside the layer [Fig. $\left.3(\mathrm{~b})\right]$. The turbulence is, however, significantly reduced in Fig. 3 (d) with the layer formation.

Frequency-integrated amplitudes over the broadband turbulent fluctuations from the X-ray and ELA array detectors at various radii are summarized in Figs. 2 (e) and 3 (e), respectively. Filled and open circles in Figs. 2 (e) and 3 (e) correspond to the cases with and without the energeticelectron-layer formation, respectively. As one can see in both figures, a significant reduction of the turbulent fluctuations is attained in and outside the energetic-electron layer $\left(5<r_{c}<10 \mathrm{~cm}\right)$. From these data, it appears that, even in the presence of the layer, there exists a high level of turbulent fluctuations in the core region $\left(r_{c}<4 \mathrm{~cm}\right)$, while a 


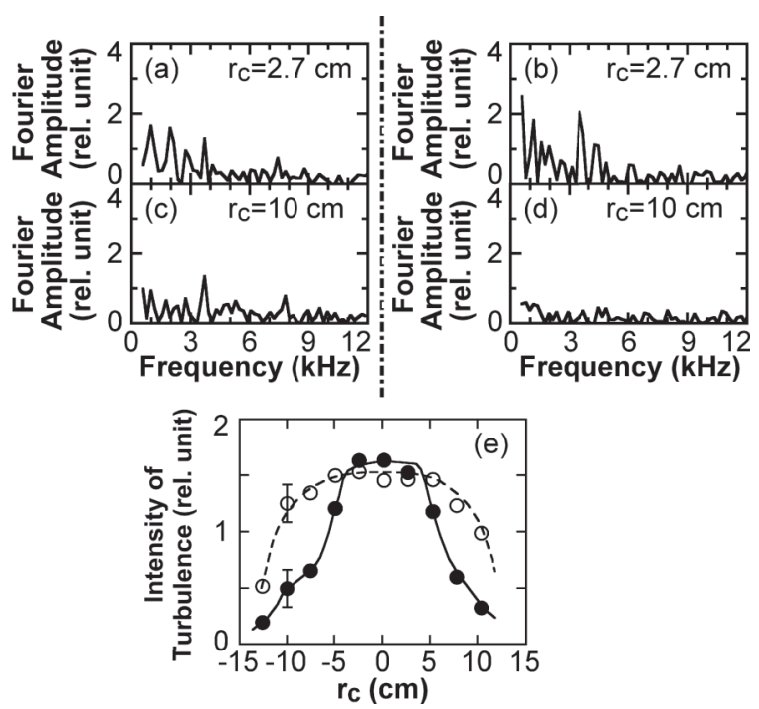

Fig. 2 (a)-(d) Fourier amplitudes of the central-cell x-ray signals are plotted at various $r_{c}$ values. The data sets are obtained in the absence [(a), (c)] and presence [(b), (d)] of the cylindrical energetic-electron layer [Fig. 1 (a)]. Frequency-integrated amplitudes over the broadband turbulent fluctuations from the x-ray detectors are summarized in (e). Solid and open circles correspond to the cases with and without the layer formation, respectively. A significant reduction in turbulence is attained in the energetic-electron layer and outside the layer (5 $\left.<r_{c}<10 \mathrm{~cm}\right)$.

quiet regime with a low level of fluctuation is observed in and outside the layer $\left(5<r_{c}<10 \mathrm{~cm}\right)$.

In Fig. 4 (a), the solid and dashed curves show $\Phi_{C}$ profiles in the presence and absence of the layer formation, respectively. In the presence and absence of the layer, the radial profile data from the central-cell HIBP and (west) IES arrays are plotted with filled and open circles, respectively. As described above, the loss of the energetic electrons leads to create a positively bumped potential enhancement located in the cylindrical layer with $E_{r}$ shear formation. Such a bumped profile of $\Phi_{C}$ results in changes of the signs of the gradient $\Phi_{C}$ and $E_{r}$ at the $\Phi_{C}$ peaked location, $r_{c} \approx 7 \mathrm{~cm} \equiv r_{p}$. This means the direction reversal of the $\boldsymbol{E}_{r} \times \boldsymbol{B}$ azimuthal $\theta$ drift flow across $r_{c}=r_{p}$; that is, the oppositely directed $\boldsymbol{E}_{r} \times \boldsymbol{B}$ sheared drift flows separate plasmas into two regions at $r_{c}=r_{p}$ from the viewpoint of actual plasma motions in $\theta$.

This feature is found in behaviors of the radial plots of the $E_{r}$ shear [Fig. 4 (b) dashed curves] and the dynamic vorticity $W$ [Fig. 4 (b) solid curves]. As shown in [3], $W$ plays a role of the canonical momentum of such rotational motion in magnetized plasmas with non-uniform density. It provides a natural generalization of the vorticity vector $\boldsymbol{w} \equiv \nabla \times \boldsymbol{V}$, which is well-known as a canonical momentum and as a measure of velocity shear in dynamics of incompressible fluids. The z-component of normalized dynamic vorticity $W \equiv\left[\nabla \times\left(n \boldsymbol{V}_{E}\right)\right]_{z} / n_{0}$ (where $n_{0}$ is the on-axis den-
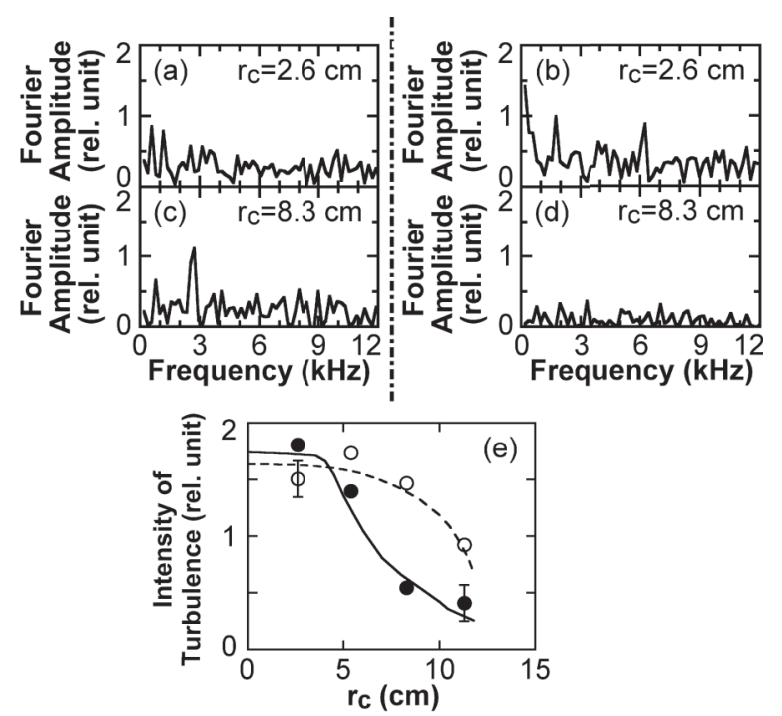

Fig. 3 (a)-(d) Fourier amplitudes of ion currents from the ELA arrays are plotted. In comparison with the data in Fig. 2, similar data sets are obtained in the absence [(a), (c)] and presence [(b), (d)] of the cylindrical energetic-electron layer. Frequency-integrated amplitudes over the broadband turbulent fluctuations from the ELA arrays are summarized in (e). Similar characteristic features of radial reduction in turbulence are observed.
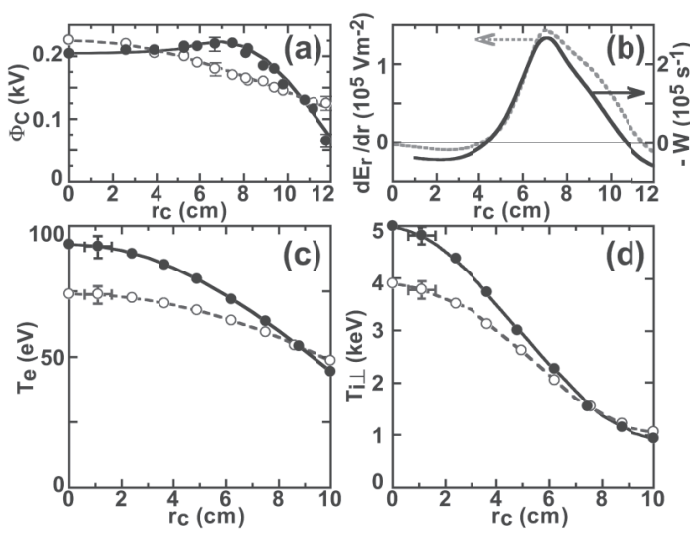

Fig. 4 (a) Central-cell potential data from HIBP and IES arrays are plotted with filled and open circles, respectively, along with solid and dashed fitting curves for the presence and absence of the energetic-electron layer, respectively. (b) $E_{r}$ shear (dashed curves) and dynamic vorticity $W$ (solid curves) are plotted in the presence of the energetic-electron layer.Solid and dashed curves show (c) $T_{e}$ and (d) $T_{i}$ profiles from x-ray and charge-exchanged particle analyses in the presence and absence of the energetic electron layer, respectively. Steep gradients in both $T_{e}$ and $T_{i}$ in a large $W$ regime along with rather flat and higher $T_{e}$ and $T_{i}$ in the inner region surrounded by the layer are found.

sity) is chosen to characterize $\boldsymbol{E} \times \boldsymbol{B}$ velocity $\left(\boldsymbol{V}_{E}\right)$ shear in our experiments $[5,6]$. In the case with the energetic electron layer, the $\Phi_{C}$ bump in Fig. 4 (a), provides significant bumps of $d E_{r} / d r_{c}$ and $W$ [Fig. 4 (b)], respectively. 
In the presence of the energetic-electron layer, the $\Phi_{C}$ profile in Fig. 4 (a), corresponds to high bumps in sheared $E_{r}$ and $W$ profiles in Fig. 4(b). The bump in $W$ is centered near $r_{c}=7 \mathrm{~cm}$, and its large-valued regime covers the above-described radii with a significant reduction of the turbulent fluctuations $\left(5<r_{c}<10 \mathrm{~cm}\right)$ in the presence of the layer. On the other hand, a high level of fluctuations in the core plasma corresponds to the weakly sheared small $W$ regime at $r_{c}<4 \mathrm{~cm}$, while a quiet regime with a low level of x-ray fluctuations in Fig. 2 (e) is observed near and outside $r_{c}=7 \mathrm{~cm}$, where $W$ becomes large with the maximum value at $r_{c}=7 \mathrm{~cm}$. Fluctuations with ELA in Fig. 3 (e) also confirm the suppression of the turbulence in the layer having high $W$ values.

Comparisons for the presence and absence of the energetic-electron layer in $T_{e}$ and $T_{i}$ profiles are shown in Figs. 4(c) and 4(d), respectively, from x-ray and charge-exchanged particle analyses. In the large $W$ regime [Fig. 4(b)], steep gradients in both $T_{e}$ and $T_{i}$ are observed. Both $T_{e}$ and $T_{i}$ increase in the core plasma region, surrounded by the localized layer having the sheared flow with the direction reversal. These findings bring us possibilities to collaborate analogical similarity studies of radial transport-barrier formation in toroidal devices [1,2]; for instance, a local heating on a magnetic surface (with some radial local losses) is a candidate for producing similar ambipolar-potential bump and barrier.

These results allow us to interpret that large-scaled turbulence typically exists in a standard regime that has a radially smooth profile of a weakly sheared plasma rotation. Stochastic vortex structures with characteristic scales comparable with the plasma radius, dominate the turbulence and causes enhanced radial heat transport. Experimental results show that the presence of a high $W$ layer leads to suppress turbulent fluctuations, and forms steep $T_{e}$ and $T_{i}$ gradients beyond the layer [Figs. 4 (c), 4 (d)]. Also, large-scaled vortices localized in the plasma core and turbulence at the plasma edge (e.g., the probe diagnostics) are almost independent of each other. This decoupling of dominant structures is an alternative example of reduction of the radial correlation length due to sheared flows.

\section{Acknowledgments}

This work was partially supported by a Grant-in-Aid for Scientific Research from Ministry of Education, Science, Sports and Culture of Japan, and partially performed under the Collaborative Research Program between NIFS and University of Tsukuba in cooperation with Japanese universities.

[1] P.H. Diamond et al., Plasma Phys. Control. Fusion 47, R35 (2005).

[2] Y. Kishimoto et al., Nucl. Fusion 40, 667 (2000).

[3] V.P. Pastukhov Plasma Phys. Reports 31, 577 (2005).

[4] T. Cho et al., Phys. Rev. Lett. 97, 055001 (2006).

[5] T. Cho et al., Nuclear Fusion 45, 1650 (2005).

[6] T. Cho et al., Phys. Rev. Lett. 94, 085002 (2005).

[7] M. Hirata et al., Trans. Fusion Sci. Tech. 47, 215 (2005).

[8] T. Cho et al., Trans. Fusion Sci. Tech. 47, 9 (2005).

[9] T. Cho et al., J. Plasma Fusion Research 80, 81 (2004).

[10] T. Cho et al., Nuclear Fusion 43, 293 (2003).

[11] T. Cho et al., Phys. Rev. Lett. 86, 4310 (2001).

[12] T. Numakura et al., Trans. Fusion Sci. Tech. 43, 222 (2003)

[13] M. Hirata et al., Nucl. Fusion 31, 752 (1991).

[14] G.I. Dimov, J. Fusion Energy 19, 87 (2001).

[15] T. Cho et al., Nuclear Fusion 41, 1161 (2001).

[16] T. Cho et al., Phys. Rev. A45, 2532 (1992).

[17] T. Cho et al., Phys. Rev. Lett. 64, 1373 (1990).

[18] M. Hirata et al., Jpn. J. Appl. Phys. 28, 96 (1989).

[19] T. Cho et al., Nucl. Fusion 28, 2187 (1988).

[20] T. Cho et al., Nucl. Fusion 27, 1421 (1987).

[21] M. Hirata et al., Nucl. Instrum. Methods B66, 479 (1992).

[22] T. Cho et al., Nucl. Instrum. Methods A348, 475 (1994).

[23] T. Cho et al., Phys. Rev. A 46, 3024 (1992).

[24] J. Kohagura et al., Phys. Rev. E56, 5884 (1997).

[25] M. Hirata et al., Rev. Sci. Instrum. 66, 2311 (1995).

[26] M. Hirata et al., Nucl. Instrum. Methods A477, 210 (2002).

[27] T. Numakura et al., Plasma Phys. Control. Fusion 45, 807 (2003).

[28] J. Kohagura et al., Rev. Sci. Instrum. 66, 2317 (1995).

[29] Y. Sakamoto et al., Rev. Sci. Instrum. 66, 4928 (1995).

[30] M. Hirata et al., Rev. Sci. Instrum. 74, 1913 (2003).

[31] M. Hirata et al., Trans. Fusion Tech. 39, 281 (2001).

[32] M. Hirata et al., Rev. Sci. Instrum. 75, 3631 (2004). 